\title{
LOMMEL FUNCTIONS IN SOME DRAG-PERTURBED PROBLEMS
}

\section{SŁAWOMIR BREITER}

Astronomical Observatory of the A. Mickiewicz University, Stoneczna 36, PL 60-286 Poznań,Poland.E-mail: breiter@phys.amu.edu.pl

and

\section{ALBERT A. JACKSON}

Lunar and Planetary Institute, 3600 Bay Area Blvd, Houston, TX 77058, USA.

Let us consider the orbital problem in which a particle is subject to the force (per unit mass)

$$
\mathbf{F}=-\frac{\mu}{r^{3}} \mathbf{r}-\frac{\alpha}{r^{2}}\left(2 \gamma \mathbf{v}_{r}+\mathbf{v}_{t}\right)
$$

The force consists of the Newtonian two body attraction term and a drag part which is linear in both components of velocity ( radial $\mathbf{v}_{r}$ and transverse $\mathbf{v}_{t}$ ). Depending on a physical interpretation of the parameters $\mu$ and $\alpha$, as well as on the particular choice of the dimensionless constant $\gamma$, the model (1) can match various two body problems with dissipation. They include the classical Poynting-Robertson effect (Robertson, 1936) with $\gamma=1$, Poynting's (1903) version with $\gamma=\frac{1}{2}$ and the two body drag cases recently studied by Mittleman and Jezewski (1982) and by Mavraganis and Michalakis (1994) under the name of Danby's drag.

Equations of motion in polar variables $r, \vartheta$ for the general model (1) can be reduced to the linear form (Breiter and Jackson 1998)

$$
\begin{aligned}
x^{2} \frac{d^{2} y}{d x^{2}}+x \frac{d y}{d x}+\left(x^{2}-\gamma^{2}\right) y & =x^{-\gamma} \\
\frac{d t}{d x} & =\frac{-\alpha^{3}}{\mu^{2} y^{2} x^{2 \gamma+1}}
\end{aligned}
$$

The reduction is achieved by means of the "generalized Robertson transformation" (Breiter and Jackson 1998)

$$
x=\frac{h}{\alpha}-\vartheta, \quad y=\frac{\alpha^{2}}{\mu r x^{\gamma}},
$$

where $h$ is the initial value of the angular momentum $r^{2} \dot{\vartheta}$. The equation of orbit (2) is a Lommel equation (Watson 1958) and its solution can be expressed in terms of the Bessel functions $J_{\gamma}(x), Y_{\gamma}(x)$ and of the Lommel function $S_{-\gamma-1, \gamma}(x)$

$$
y(x)=A J_{\gamma}(x)+B Y_{\gamma}(x)+S_{-\gamma-1, \gamma}(x),
$$


where $A, B$ are arbitrary constants. The time equation (3) is reduced to a quadrature, but it does not seem to be expressible by known special functions in a closed form.

Even without the knowledge of the explicit dependence of $x(t)$ the orbit solution (5) can lead to some interesting conclusions. Klačka and Kaufmannová (1992) observed that the eccentricity of some orbits in Poynting-Robertson problem can grow systematically. Using equations (3) and (5), and taking the limits at $x \rightarrow 0$, one can verify, that osculating eccentricity tends asymptotically to $e=1$ for all orbits in the general problem (1). The true anomaly $f$ is a fast variable only at the initial stage of the orbital evolution, when the eccentricity decreases. But once the eccentricity starts increasing, $f$ tends slowly towards the asymptotic value $f=\frac{3}{2} \pi$ (Breiter and Jackson 1998). Another interesting conclusion is that the classical solution for the Poynting-Robertson problem of Wyatt and Whipple (1950) should be used with caution. The quasi-integral $p e^{-4 / 5}$, which is the comerstone of this solution, reveals a significant secular trend during the final stage of motion.

A definite advantage of the solution (5) is its general validity for all types of motion, including capture or escape problems from open and closed orbits.

\section{References}

Breiter S., Jackson A. A.: 1998, 'Unified analytical solutions to two-body problems with drag', MNRAS, 299, 237-243.

Klačka J., Kaufmannová J.: 1992, 'Poynting-Robertson Effect: "Circular" Orbit', Earth, Moon and Planets, 59, 97-102.

Mavraganis A. G., Michalakis D. G.: 1994, 'The Two-Body Problem with Drag and Radiation Pressure', Celest. Mech. \& Dynam. Astron., 58, 393-403.

Mittleman D., Jezewski D.: 1982, 'An Analytic Solution to the Classical Two-Body Problem with Drag', Celest. Mech., 28, 401-413.

Poynting J. H.: 1903, 'Radiation in the Solar System: its Effect on Temperature and its Pressure on Small Bodies', Phil. Trans. Roy. Soc., A 202, 525-552.

Robertson H. P.: 1937, 'Dynamical Effects of Radiation in the Solar System', MNRAS, 97, 423-438. Watson G. N.: 1958,A Treatise on the Theory of Bessel Functions, Cambridge Univ. Press, Cambridge. Wyatt S. P., Whipple F. L.: 1950, 'The Poynting-Robertson Effect on Meteor Orbits', ApJ, 111, 558-565. 